\title{
Benchmarks
}

\section{Elimination of the plasmid bacterial backbone in site-directed transgenesis}

Jannik E. Jakobsen, Jacob G. Mikkelsen, and Anders L. Nielsen Department of Human Genetics, University of Aarbus, Denmark

BioTechniques 48:313-316 (April 2010) doi 10.2144/000113386

Keywords: transgenesis; FLP-recombinase; FRT site; minicircle; bacterial backbone

Supplementary material for this article is available at www.BioTechniques.com/article/113386.

For cellular and animal transgenesis, FLP- and Cre-recombinase gene capture systems are highly effective to provide stable integration of a donor plasmid carrying the transgene cassette of interest into an engineered genomic locus in a given cell line. However, in many protocols, the entire plasmid bacterial backbone is integrated along with the transgene cassette. Here, we present a very simple yet highly efficient method for excluding plasmid bacterial backbone integration. The transgene cassette, including a single FLP recognition target site, is specifically amplified by PCR, and the resulting DNA ligated into minicircles can serve as donor DNA in FLP-mediated recombination. Interestingly, the elimination of the bacterial backbone increased expression of the inserted transgene. The presented method is simple and efficient for generating transgene cassette insertions devoid of the bacterial backbone.

Site-specific recombinases represent important tools in eukaryotic transgenesis. Recombinases like FLP and Cre have been widely used to direct transgene insertion to constitutively active chromosomal locations and to eliminate transgene concatemerization (1-3). By sequential transgenesis, cells of interest are first engineered to contain a 'docking' site containing recombinase recognition sequences and a stably expressed marker gene. Such cells are used subsequently for transgene cassette insertion through site-specific recombination. In many procedures, recombination-mediated transgenesis will result in co-insertion of the plasmid bacterial backbone (BB) which can influence transgene expression and constitutes an undesired source of bacterial DNA and resistance genes for the recipient cells (4). Recently, recombinase-mediated cassette exchange was developed by relying on tandem-incompatible recombination target sites for transgene insertion (5) One advantage of recombinase-mediated cassette exchange is the possibility to exclude co-insertion of the BB. Accordingly, $\mathrm{BB}$ insertion into the recipient cell genome will depend on additional random co-integrations of the transgene vector. Random integration is unfavored compared with recombinase-mediated integration, but random co-integration of $\mathrm{BB}$ sequences is observed if cells are prone to random integrations (6). A limitation of the use of recombinase-mediated cassette exchange for $\mathrm{BB}$ removal is the requirement for tandem recombination sites, which excludes widely used transgenesis vectors, cell lines, and animal models possessing only a single recombination site. Generation of donor minicircles in bacteria represents an alternative strategy for production of BB-deficient DNA substrates for transgenesis $(7,8)$. This approach is complicated by the requirements for specific bacterial strains and vector designs. We sought a simple, time-saving, and general alternative method to avoid integrating the $\mathrm{BB}$. Here, we present a very simple yet highly efficient method in which a transgene expression cassette without $\mathrm{BB}$ is amplified by PCR and the resulting DNA is ligated into minicircles to serve as donor DNA in FLP-mediated recombination.
The transgene donor plasmid used in our analysis is a modified version of pcDNA5/FRT (Invitrogen, Carlsbad, CA, USA) (FRT: FLP recognition target) in which the red fluorescent protein (DsRed) gene was inserted. This vector, designated pcDNA5/FRT-red (Figure 1A), also contains the Hygromycin resistance gene that lacks a start methionine (Invitrogen). To generate recombinase substrate DNA without $\mathrm{BB}$, the DsRed/Hygromycin transgene cassette was amplified by PCR (see Supplementary Material for details) using primers with terminal BglII sites recognizing sequences immediately upstream of the CMV promoter and downstream of the Hygromycin resistance gene polyadenylation signal, respectively (primer locations indicated in Figure 1A). The PCR sample was digested with $D p n$ I to remove template DNA. For generation of minicircles, DNA was cleaved with $B g l$ II and used in a T4 DNA ligation reaction (New England BioLabs, Ipswich, MA, USA) favoring intramolecular ligation. The minicircle DNA is schematically illustrated in Figure 1B.

For site-directed transgenesis, we used different cell lines. The FLP-In T-Rex HEK293 and BHK cell lines have insertion of a single FRT site linked to the Zeocin resistance gene (Invitrogen). Upstream from the FRT site is a methionine codon conferring the start codon to the Hygromycin resistance gene of the donor plasmid or minicircle after FLP-mediated recombination. Additionally, we have generated a HEK293-derived cell line, HEK293-6F with 6 insertions of the Sleeping Beauty transposon SBT/SV40-FGIP (Figure 1C). SBT/SV40-FGIP includes a FRT-EGFP fusion gene driven by the SV40 promoter (9) and a methionine codon located upstream from the FRT site conferring the start codon to the Hygromycin resistance gene of the donor plasmid or minicircle after FLP-mediated recombination.

For the characterization of transgenesis using minicircle donor DNA, we initially used the HEK293-6F cell line since the presence of multiple FRT sites will allow simultaneous examination of several transgenesis events in each cell clone. For FLP-mediated transgenesis, $0.5 \mu \mathrm{g} \mathrm{cDNA5/FRT-red} \mathrm{(or} \mathrm{a}$ similar amount of minicircle DNA) was transfected into HEK293-6F cells. The DNA was co-transfected with $5.5 \mu \mathrm{g}$ either FLP-recombinase expression vector pOG44 (Invitrogen) 
A

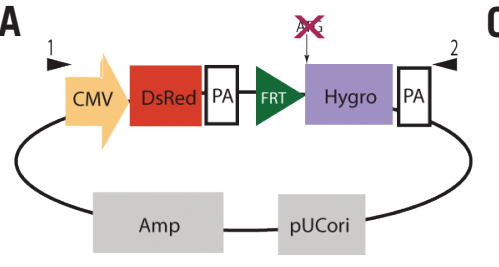

FLP donor plasmid pcDNA5/FRT-red

B

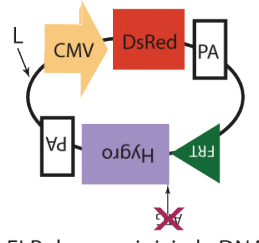

FLP donor minicircle DNA

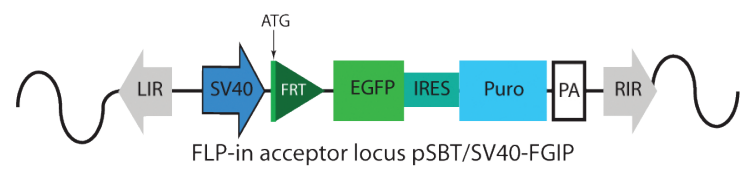

FLP-in acceptor locus PSBT/SV40-FGIP

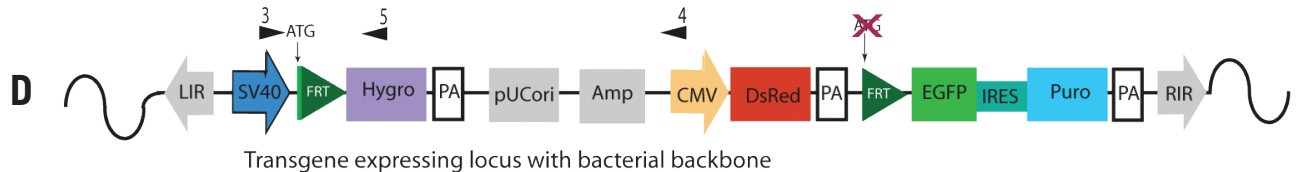

Transgene expressing locus with bacterial backbone

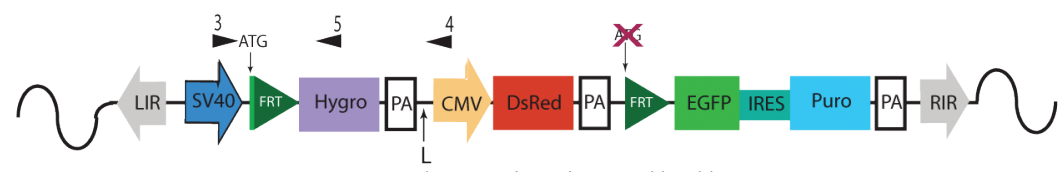

Transgene expressing locus without bacterial backbone

Figure 1. FLP-mediated transgenesis using bacterial backbone (BB)-deficient DNA substrates. (A) Schematic presentation of pcDNA5/FRT-red plasmid used for FLP-mediated transgenesis. Primer locations for the PCR fragment to generate the minicircle are indicated on the plasmid map. The absence of a functional ATG start codon for the Hygromycin resistance gene is indicated by the red $\times$. Amp, ampicillin resistance gene; pUCori, plasmid replication origin; pA, polyadenylation signal; Hygro, Hygromycin B resistance gene; CMV, cytomegalovirus promoter; DsRed, red fluorescent protein; FRT, FLPrecombination target site. (B) Schematic presentation of the minicircle donor DNA used for FLP-mediated transgenesis. The site of self-ligation in the minicircle DNA is indicated by " $\mathrm{L}$ " and an arrow. (C) Schematic overview of the acceptor 'docking' locus for FLP-recombination present in HEK293-6F cells and the FLP recombinase expression vector. The left and right inverted repeat motifs (LIR and RIR) represent the terminal sequences of the transposon vector used for the primary HEK293 transgenesis. IRES, internal ribosomal entry site; EGFP, enhanced green fluorescent protein; Puro, puromycin resistance gene; SV40; Simian virus 40 promoter. Note that the HEK293 and BHK FLP-In cell lines do not include the LIR and RIR sequences together with the EGFP-IRES-Puromycin module and instead harbor a Zeocin resistance gene (Invitrogen). (D) Schematic overview of transgene insertion after FLP-mediated recombination in HEK293-6F cells, showing the transgene-expressing locus after transgenesis with the pcDNA5/FRT-red vector (upper) and the transgene-expressing locus after transgenesis with BB-deficient minicircle (lower). Positions of primers 3, 4, and 5 used for delineation of the recombination events and transgene cassette integrity are indicated above the schematic drawings.

or pUC19 as a negative control. The next day, cells were diluted $10 \times$ and after 2 days, Hygromycin was added. Resistant colonies were formed only from cells co-transfected with pOG44 and pcDNA5/FRT-red or minicircle DNA, verifying that transgenesis was FLP-dependent. The number of colonies was similar for the two DNA donors (30-55 colonies per $10^{5}$ transfected HEK293-6F cells). Genomic DNA was purified from colonies independent of the fluorescence status to ensure nonbiased analysis of targeted integration events. Recombination into the FRT site was examined by PCR screening of genomic DNA using primers recognizing the $S V 40$ promoter and the Hygromycin resistance gene (primer combination 3 and 5 indicated in Figure 1D). A 300-bp fragment was expected after recombination into the FRT site and indeed, such a PCR product was observed from all examined genomic DNA samples (Figure 2A). Sequencing confirmed precise recombination (data not shown). This clearly supports that minicircle DNA devoid of $\mathrm{BB}$ can be used for FLP-mediated transgenesis. We addressed $\mathrm{BB}$ deletion by PCR using primers located in the SV40 promoter and upstream from the
CMV promoter (primer combination 3 and 4 in Figure 1D). In all six examined DNA samples from minicircle transgenesis, a $1.7-\mathrm{Kb}$ fragment was detected which represents the expected size for a BglII-site ligation and, accordingly, an absence of the BB (Figure 2B). The lack of $\mathrm{BB}$ was confirmed by sequencing (data not shown). Southern blot analyses showed DNA rearrangements in clone \#2 from the minicircle donor (Supplementary Figure S1A). Clone \#3 from the minicircle donor indicated existence of transgene concatemerization (Supplementary Figure S1A).

Next, we measured transgene expression after minicircle-mediated transgenesis by analyzing Hygromycin-resistant colonies for expression of DsRed and EGFP. Note that since HEK293-6F cells have multiple insertions of SBT/SV40-FGIP, which express EGFP, correctly targeted recombination does not necessarily result in the disappearance of EGFP expression as this requires that all EGFP-expressing loci are targeted simultaneously. The number of EGFP and DsRed fluorescent cell clones was similar using minicircle or plasmid donor DNA, and the homogeneity of DsRed expression in the cell clones was independent of the type of donor DNA used (data not shown). We note that clone \#2 from the minicircle transgenesis which contains DNA rearrangements lacked DsRed expression and was not representative of minicircle transgenesis as all other obtained colonies were DsRed positive.

To substantiate the usability of the minicircle approach, we next examined FLP-mediated site-directed recombination in cell lines harboring a single FRT site. In the commercially available FLP-In T-Rex HEK293 (HEK293-1F) and $\mathrm{BHK}(\mathrm{BHK}-1 \mathrm{~F})$ cell lines (Invitrogen), we obtained similar numbers of Hygromycin resistance colonies by FLP-mediated transgenesis using either plasmid or minicircle donor DNA (10-20 colonies per $10^{5}$ transfected cells). The colony-forming capacity in these cells was $\sim 3 \times$ lower than in HEK293-6F cells, which could be due to the decreased number of FRT sites. In the single-FRT cell lines, control experiments excluding the FLP expression plasmid in the transgenesis showed, as expected, a very low colony-forming capacity $\left(0-1\right.$ colonies per $10^{5}$ transfected cells). PCR analysis of colonies verified the correct targeted insertion of transgenes and fluorescence microscopy showed similar frequencies of DsRed- 


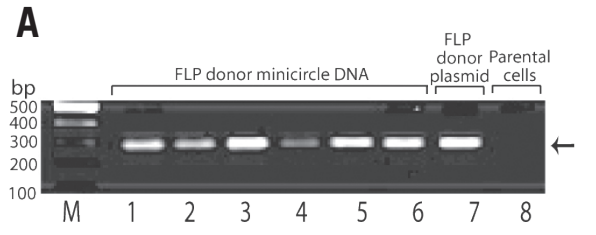

B

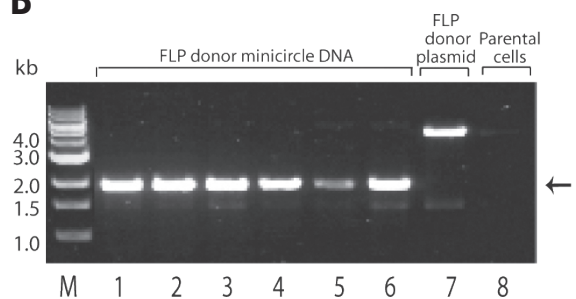

C

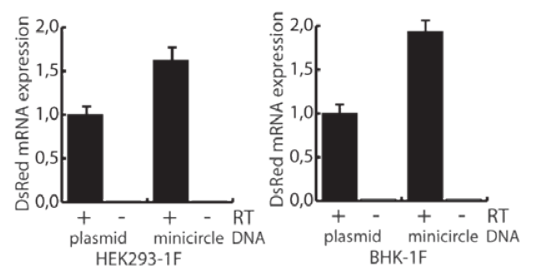

Figure 2. Characterization of minicircle transgenesis. (A) Confirmation of FLP-mediated recombination of minicircle DNA into the FRT site of HEK293-6F cells. DNA was extracted and examined from six colonies for minicircle DNA transgenesis (lanes 1-6), from one representative colony for pcDNA5/FRT-red transgenesis (lane 7), and from the parental cells (lane 8). Transgenesis into the FRT site was monitored by PCR using primer combination 3 and 5 . The 300-bp band resulting from recombination into the FRT site is indicated by an arrow. A 100-bp marker was loaded in the leftmost lane. (B) Confirmation of ligation/circle closure in transgenic clones obtained from minicircle transgenesis into the FRT site of HEK293-6F cells. DNA samples, as described in Figure 1, were analyzed by PCR using primer combination 3 and 4 . The 1.7-kb fragment resulting from FLP-mediated transgenesis of minicircles ligated in the Bgll sites is indicated by an arrow. The 3.8-kb fragment in lane 13 represents integration of the plasmid donor pcDNA5/FRT-red containing the BB. A 1-kb marker was loaded in the leftmost lane. (C) The BB influences transgene expression in the single-FRT site HEK293 and BHK cell lines. The expression level of DsRed mRNA was measured by qRT-PCR. Mock cDNA synthesis reactions lacking addition of reverse transcriptase (RT) were used as controls. Melting curve analysis and gel electrophoresis were used to confirm PCR products. The qRT-PCR reactions were performed on $\mathrm{CDNA}$ derived from a single colony pool (20 colonies) for each donor DNA. Technical triplicates were performed and used for calculating mean values and standard deviations. The expression level of DsRed was normalized to the expression level of GAPDH and the DsRed expression level for the plasmid donor cell sample further normalized to 1 . Detailed information concerning the different PCR reactions is available in the Supplementary Materials. expressing colonies using plasmid and minicircle donor DNA, and that the homogeneity of DsRed expression within colonies was similar (data not shown). To examine the effect of excluding the BB for transgene expression, the level of DsRed mRNA was measured by quantitative reverse transcription PCR (qRT-PCR) performed on RNA extracted from one colony pool (20 colonies) for each DNA donor (see Supplementary Materials). The qRT-PCR analysis showed that the $\mathrm{BB}$ had a negative effect on the order of $1.5-2 \times$ for the DsRed mRNA expression level (Figure 2C).

We here demonstrate a simple and universal alternative protocol to eliminate $\mathrm{BB}$ co-integration in sitedirected recombination. Any donor plasmid can be applied and easily converted into minicircle DNA. We note that an alternative to the PCR amplification of the transgene cassette is the use of restriction enzymes flanking the transgene cassette and performing re-ligation to establish minicircles. This procedure allows generation of $\mathrm{BB}$-free donor DNA in circumstances where PCR amplification is undesirable. We also note that the ligation step can be excluded for $\mathrm{BB}$-free transgenesis, though this results in an increased frequency of transgene insertions with rearrangements (see Supplementary Figure S2). Exclusion of the BB resulted in an increase in DsRed mRNA expression in the single FRT insertion cell lines that we examined. This supports a negative effect of the $\mathrm{BB}$ on transgene expression. We note that the cell lines used for targeted transgenesis were all selected for a high and stable expression of the ectopic resistance gene in the initial generation of FRT-site insertions. Thus, the effect of the BB for transgene expression might be diminished by the selected loci and it will be interesting to examine the effect of the BB for expression in transgene insertions not pre-selected for continuously transcriptionally active chromatin. We conclude that the described minicircle approach to achieve BB-free transgenesis constitutes a relatively secure, effective, and simple alternative to existing methods.

\section{Acknowledgments}

We thank Marianne Johansen and Tina Fuglsang Daugaard for technical assistance. J.E.J. is the recipient of a Ph.D. fellowship from the Medical Faculty,
University of Aarhus, Denmark. The work by J.E.J. was carried out in the laboratory of Arne Lund Jørgensen of the Department of Human Genetics at the University of Aarhus. This project was supported by the Danish Research Council (FSS) and the NovoNordic Foundation.

\section{Competing interests}

The authors declare no competing interests.

\section{References}

1.Akopian, A. and W. Marshall Stark. 2005. Site-specific DNA recombinases as instruments for genomic surgery. Adv. Genet. 55:1-23.

2.Kolb, A.F. 2002. Genome engineering using site-specific recombinases. Cloning Stem Cells 4:65-80.

3. Branda, C.S. and S.M. Dymecki. 2004. Talking about a revolution: The impact of site-specific recombinases on genetic analyses in mice. Dev. Cell 6:7-28.

4.Etchberger, J.F. and O. Hobert. 2008. Vector-free DNA constructs improve transgene expression in C. elegans. Nat. Methods 5:3.

5. Wirth, D., L. Gama-Norton, P. Riemer, U. Sandhu, R. Schucht, and H. Hauser. 2007. Road to precision: recombinasebased targeting technologies for genome engineering. Curr. Opin. Biotechnol. 18:411419.

6. Louwerse, J.D., M.C. van Lier, D.M. van der Steen, C.M. de Vlaam, P.J. Hooykaas, and A.C. Vergunst. 2007. Stable recombinasemediated cassette exchange in Arabidopsis using Agrobacterium tumefaciens. Plant Physiol. 145:1282-1293.

7. Chen, Z.Y., C.Y. He, and M.A. Kay. 2005. Improved production and purification of minicircle DNA vector free of plasmid bacterial sequences and capable of persistent transgene expression in vivo. Hum. Gene Ther. 16:126-131.

8. Mayrhofer, P., M. Blaesen, M. Schleef and W. Jechlinger. 2008. Minicircle-DNA production by site specific recombination and protein-DNA interaction chromatography. J Gene Med. 10:1253-1269.

9. Moldt, B., N.H. Staunstrup, M. Jakobsen, R.J. Yanez-Munoz, and J.G. Mikkelsen. 2008. Genomic insertion of lentiviral DNA circles directed by the yeast Flp recombinase. BMC Biotechnol. 8:60.

Received 24October 2008; accepted 16 February 2010.

Address correspondence to Anders Lade Nielsen, Department of Human Genetics, The Bartholin Building, University of Aarhus, DK-8000 Aarhus C, Denmark. e-mail: aln@ humgen.au.dk 